\title{
A megahertz bandwidth dual amplifier for driving piezoelectric actuators and other highly capacitive loads
}

\author{
Andrew J. Fleming \\ The School of Electrical Engineering and Computer Science, The University of Newcastle, Callaghan 2308, \\ Australia
}

(Received 13 August 2009; accepted 31 August 2009; published online 5 October 2009)

\begin{abstract}
Due to their high stiffness, small dimensions, and low mass, piezoelectric stack actuators are capable of developing large displacements over bandwidths of greater than $100 \mathrm{kHz}$. However, due to their large electrical capacitance, the associated driving amplifier is usually limited in bandwidth to a few kilohertz or less. In this paper the limiting characteristics of piezoelectric drives are identified as the small-signal bandwidth, output impedance, cable inductance, and power dissipation. A new dual amplifier is introduced that exhibits a small-signal bandwidth of $2 \mathrm{MHz}$ with a $100 \mathrm{nF}$ capacitive load. The dual amplifier is comprised of a standard high-voltage amplifier combined with a fast low-voltage amplifier to improve performance at higher frequencies. Experiments demonstrate a $300 \mathrm{kHz}$ sine wave of $20 \mathrm{Vp}$-p amplitude being applied to a $100 \mathrm{nF}$ load with negligible phase delay and a peak-to-peak current of $3.8 \mathrm{~A}$. With a voltage range of $200 \mathrm{~V}$ and peak current of $1.9 \mathrm{~A}$ a standard amplifier would require a worst-case power dissipation of $380 \mathrm{~W}$. However, the dual-amplifier arrangement has a worst-case power dissipation of only $30 \mathrm{~W}$. The penalty is reduced range at high frequencies and slower operation from the high-voltage stage. (C) 2009 American Institute of Physics. [doi:10.1063/1.3234261]
\end{abstract}

\section{INTRODUCTION}

Piezoelectric transducers are the actuator of choice in applications requiring precision motion and force control. They are compact, light weight, and high in stiffness. These properties permit high mechanical resonance frequencies, typically in the tens or hundreds of kilohertz.

Many applications utilize the high-speed and precision offered by piezoelectric actuators. Examples include nanofabrication systems, ${ }^{1}$ high-speed micromechanical systems, ${ }^{2}$ scanning probe microscopes (SPMs), ${ }^{3,4}$ and vibration control systems. ${ }^{5,6}$

Both SPMs and nanofabrication systems use piezoelectric tube or stack based scanners for sample positioning and probe control. With increasing SPM imaging speed and nanofabrication throughput, greater demands are placed on the bandwidth of the positioning stages. ${ }^{7-10}$ These demands have necessitated the use of monolithic piezoelectric stack actuators to achieve the required stiffness and resonance frequency. Unfortunately, due to the high capacitance of these actuators, the system bandwidth is usually limited by driving electronics. A number of wide bandwidth high-voltage amplifier designs have been previously presented; ${ }^{11,12}$ however, these are designed for electro-optic modulators with capacitances on the order of $100 \mathrm{pF}$. There are presently no $\mathrm{MHz}$ bandwidth high-voltage amplifier designs available to drive load capacitances in the order of $100 \mathrm{nF}$ or greater.

The first contribution of this paper is to identify the limitations of an electronic drive when loaded with a piezoelectric actuator or other highly capacitive load. In Sec. II, these limitations are established to be the finite output impedance, cable inductance, small-signal bandwidth, current limit, and power dissipation of the drive.
To circumvent the limitations identified, a new amplifier is proposed in Sec. III. The proposed dual amplifier comprises a standard high-voltage amplifier and secondary lowvoltage amplifier to increase performance at high frequencies. Experimental results in Sec. IV demonstrate a $2 \mathrm{MHz}$ small-signal bandwidth with $100 \mathrm{nF}$ load capacitance.

\section{BANDWIDTH LIMITATIONS}

\section{A. Passive bandwidth limitations}

Two major causes of bandwidth limitation are the amplifiers high-frequency output impedance and the inductance of cables and connectors. Note that the high-frequency output impedance is not to be confused with the dc output impedance typically quoted by amplifier manufacturers. Consider the electrical circuit shown in Fig. 1 where a voltage source is connected to a piezoelectric actuator. The actuator is modeled as a capacitance $C_{p}$ in series with a strain dependent voltage source $V_{p}$. The resistance $R_{s}$ and inductance $L$ are the source impedance and cable inductance respectively.

The cable inductance per meter $L_{m}$ can be calculated from the characteristic impedance $Z_{0}$ and the capacitance per meter $C_{m}$ using the equation ${ }^{13}$

$$
L_{m}=Z_{0}^{2} C_{m} \text {. }
$$

The inductance of standard RG-58 coaxial cable is 250 $\mathrm{nH} / \mathrm{m}$, this is lower than typical speaker cable which has an inductance of around $600 \mathrm{nH} / \mathrm{m}$. Both are commonly used as interconnects between amplifiers and actuators.

The amplifier source impedance refers to the highfrequency output impedance of the amplifier. In commercially available amplifiers, $R_{s}$ is typically between 10 and 


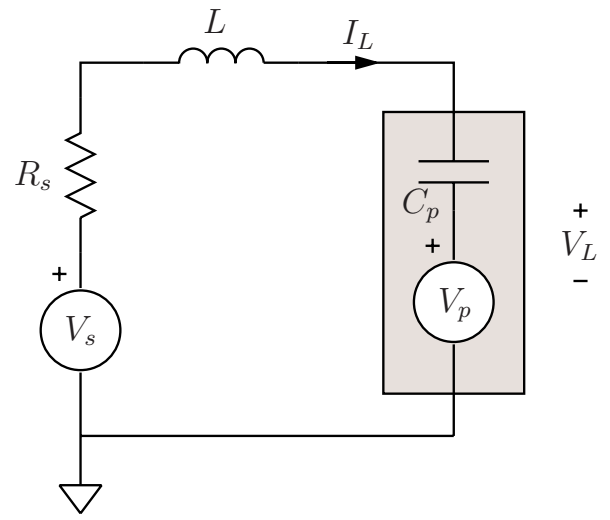

FIG. 1. (Color online) A voltage source $V_{s}$ driving a piezoelectric load. The actuator is modeled by a capacitance $C_{p}$ and strain dependent voltage source $V_{p}$. The resistance $R_{s}$ and inductance $L$ are the output impedance and cable inductance, respectively.

$100 \Omega$. When considering the effects of both output impedance and cable inductance, the transfer function from source voltage $V_{s}$ to load voltage $V_{L}$ is

$$
\frac{V_{L}(s)}{V_{s}(s)}=\frac{\frac{1}{L C_{p}}}{s^{2}+\frac{R_{s}}{L} s+\frac{1}{L C_{p}}},
$$

where $s$ is the Laplace transform variable. This is a unitygain second-order resonant low-pass filter with resonance frequency $f_{r}$ and damping ratio $\xi$ defined by

$$
f_{r}=\frac{1}{2 \pi \sqrt{L C_{p}}}, \quad \xi=\frac{R_{s} \sqrt{L C_{p}}}{2 L} .
$$

If inductance is neglected, the first-order cutoff frequency resulting from the source resistance is

$$
f_{c}=\frac{1}{2 \pi R_{s} C_{p}} .
$$

In Table I the first-order cutoff frequency and resonance frequency is tabulated for a range of typical values for $R_{s}$, $C_{p}$, and $L$. Clearly, the output impedance is of primary con-

TABLE I. Bandwidth limitation imposed by source impedance (a) and cable inductance (b).

(a) Bandwidth due to $R_{s}$

\begin{tabular}{cccc}
\hline & \multicolumn{3}{c}{$C_{p}$} \\
\cline { 2 - 4 }$(\Omega)$ & $100 \mathrm{nF}$ & $1 \mu \mathrm{F}$ & $10 \mu \mathrm{F}$ \\
\hline 1 & $1.6 \mathrm{MHz}$ & $160 \mathrm{kHz}$ & $16 \mathrm{kHz}$ \\
10 & $160 \mathrm{kHz}$ & $16 \mathrm{kHz}$ & $1.6 \mathrm{kHz}$ \\
100 & $16 \mathrm{kHz}$ & $1.6 \mathrm{kHz}$ & $160 \mathrm{~Hz}$
\end{tabular}

(b) Resonance frequency due to $L$

\begin{tabular}{cccc} 
& \multicolumn{3}{c}{$C_{p}$} \\
\cline { 2 - 4 }$(\mathrm{nH})$ & $100 \mathrm{nF}$ & $1 \mu \mathrm{F}$ & $10 \mu \mathrm{F}$ \\
\hline 25 & $3.2 \mathrm{MHz}$ & $1 \mathrm{MHz}$ & $320 \mathrm{kHz}$ \\
250 & $1 \mathrm{MHz}$ & $320 \mathrm{kHz}$ & $100 \mathrm{kHz}$ \\
2500 & $320 \mathrm{kHz}$ & $100 \mathrm{kHz}$ & $32 \mathrm{kHz}$ \\
\hline \hline
\end{tabular}

cern. This is because the bandwidth imposed by source impedance is inversely proportional to both resistance and capacitance. Reductions in both of these parameters can achieve significant bandwidth improvements. Alternatively, the resonance frequency is inversely proportional to both $\sqrt{ } L$ and $\sqrt{C_{p}}$, so a fourfold reduction in $L$ or $C_{p}$ is required to double the bandwidth.

Although it is difficult to achieve improvements in resonance frequency, all effort should be expended in doing so as a lightly damped resonance in the transfer function is highly undesirable. In addition to oscillation induced by wide bandwidth input signals, the gain peaking and phase lag can severely limit the performance of feedback control systems in which the amplifier and actuator are enclosed.

\section{B. Amplifier bandwidth}

The most obvious bandwidth limitation is the smallsignal bandwidth of the amplifier. In commercial devices, this can range from a few hundred Hertz to over $100 \mathrm{kHz}$. Unfortunately these figures are load dependent. The highly capacitive impedance and resonant nature of piezoelectric loads introduces phase lag into the feedback path. This reduces bandwidth, decreases phase-margin and can lead to instability. For standard voltage-feedback amplifiers with dominant pole compensation, a rough rule of thumb is that closed-loop bandwidth cannot exceed one tenth the cutoff frequency of the pole formed by the amplifiers output impedance $R_{s}$ and load capacitance $C_{p}$. Typical frequencies for this pole are shown in Table I (a). Thus, with standard voltagefeedback amplifiers, the dominant limitation is the output pole. To improve performance, this pole will either have to be increased in frequency, or removed from the closed-loop transfer function of the amplifier, or both.

Further bandwidth restrictions are imposed by the maximum slew rate of the amplifier. This is the maximum rate at which the output voltage can change and is usually expressed in volts per microsecond. For sinusoidal signals, the maximum frequency is

$$
f^{\mathrm{max}, \sin }=\frac{\mathrm{SR}}{V_{\mathrm{p}-\mathrm{p}} \pi},
$$

where $V_{\mathrm{p}-\mathrm{p}}$ is the peak-to-peak voltage and $\mathrm{SR}$ is the slew rate. The triangular signals used in scanning systems require a lesser slew rate and thus have a slightly higher maximum frequency of

$$
f^{\text {max,tri }}=\frac{\text { SR }}{V_{\mathrm{p}-\mathrm{p}} 2} .
$$

If a $300 \mathrm{kHz}$ sine wave is to be reproduced with an amplitude of $20 \mathrm{Vp}$-p, the required slew rate is $19 \mathrm{~V} / \mu \mathrm{s}$. This value is proportional to both frequency and amplitude. Although slew rate limitations can be critical when considering resistive or inductive loads, when dealing with capacitive loads, the current limit is usually exceeded well before the slew rate limit. This is discussed in the following subsection. 
TABLE II. Minimum current requirements for a $10 \mathrm{~V}$ sinusoid.

\begin{tabular}{cccc}
\hline \hline & \multicolumn{3}{c}{$C_{p}$} \\
\cline { 2 - 4 }$f$ & $100 \mathrm{nF}$ & $1 \mu \mathrm{F}$ & $10 \mu \mathrm{F}$ \\
\hline $30 \mathrm{~Hz}$ & $0.19 \mathrm{~mA}$ & $1.9 \mathrm{~mA}$ & $19 \mathrm{~mA}$ \\
$3 \mathrm{kHz}$ & $19 \mathrm{~mA}$ & $190 \mathrm{~mA}$ & $1.9 \mathrm{~A}$ \\
$300 \mathrm{kHz}$ & $1.9 \mathrm{~A}$ & $19 \mathrm{~A}$ & $190 \mathrm{~A}$ \\
\hline \hline
\end{tabular}

\section{Current and power limitations}

Neglecting the piezoelectric strain voltage, i.e., when driving the actuator off-resonance, the current delivered to a piezoelectric actuator is approximately

$$
\begin{aligned}
& I_{L}(s)=V_{L}(s) C_{p} s, \\
& =C_{p} \frac{d V_{L}(t)}{d t} .
\end{aligned}
$$

For sinusoidal signals, the maximum positive and negative current is equal to

$$
I_{L}^{\max , \sin }= \pm V_{\mathrm{p}-\mathrm{p}} \pi f C_{p} .
$$

For triangular signals, the maximum current is

$$
I_{L}^{\max , \text { tri }}=V_{\mathrm{p}-\mathrm{p}} 2 f C_{p} .
$$

Examples of current requirements for different load capacitances and frequencies are shown in Table II.

When designing the current limit of an amplifier, the key parameter is the maximum allowable power dissipation. The power dissipation in the output stage of a linear amplifier is equal to the product of current through, and voltage across the power transistors. That is, the instantaneous power dissipation $P_{d}$ is

$$
P_{d}=I_{L}\left(V_{\text {rail }}-V_{L}\right)
$$

where $V_{\text {rail }}$ the is the internal power supply voltage.

If an amplifier is designed to tolerate output short circuits, i.e., $V_{L}=0$, the maximum power dissipation sets the limit on maximum allowable output current. Foldback current limiting can increase maximum output current but is not suitable for capacitive loads where maximum current can be required with low or zero output voltage.

\section{DUAL AMPLIFIER}

\section{A. Circuit operation}

High voltage amplifiers cannot achieve bandwidths over $100 \mathrm{kHz}$ with large capacitive loads for two main reasons. First, the output impedance of high-voltage output stages is typically greater than $10 \Omega$, this limits the small-signal bandwidth to the values shown in Table I (a). Second, the required current limit for even a $10 \mathrm{Vpk}$ sinusoidal voltage is $0.63 \mathrm{~A}$ at $100 \mathrm{kHz}$. If the amplifier supply voltage is $200 \mathrm{~V}$, a current limit of $0.63 \mathrm{~A}$ implies a worst-case (short-circuit) power dissipation of $126 \mathrm{~W}$, which is impractically high. For these reasons, high-voltage amplifiers driving capacitive loads of $100 \mathrm{nF}$ or greater are usually restricted in bandwidth to around $10 \mathrm{kHz}$.

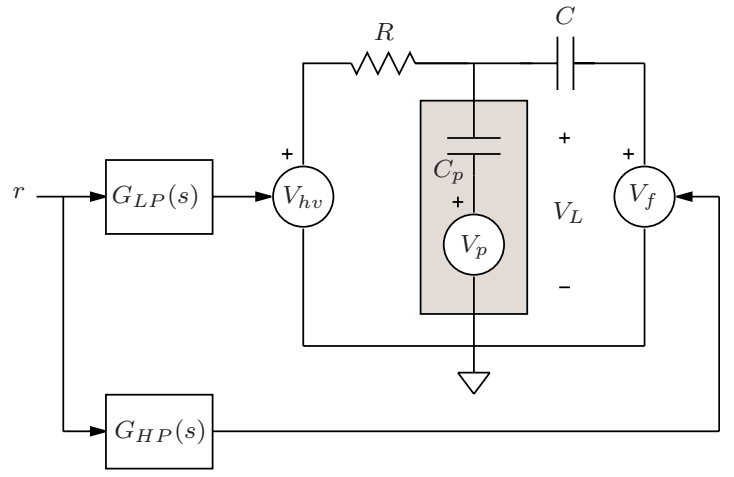

FIG. 2. (Color online) High-speed dual amplifier. The reference signal $r$ is applied simultaneously to a slow high-voltage amplifier $V_{\mathrm{hv}}$ and a fast lowvoltage amplifier $V_{f}$. The two amplifiers are coupled to the load through the resistance $R$ and $C$, respectively.

In this work, a new amplifier is presented that alleviates the problems of high-voltage amplifiers by adding an auxiliary low-voltage stage to improve output impedance and drop power dissipation at high frequencies. A schematic diagram of the dual amplifier is shown in Fig. 2. Essentially the dual amplifier comprises two amplifiers, a standard highvoltage amplifier $V_{\mathrm{hv}}$ and a fast low-voltage amplifier $V_{f}$. The low-voltage stage drives the actuator at high frequencies but with reduced range. Due the lower supply voltage (say \pm 15 V) the low-voltage stage dissipates less power and can use bipolar transistors under heavy forward bias to provide an output impedance in the milliohm range.

The two amplifiers are coupled to the load through the resistor $R$ and capacitor $C$. This network ensures that the load voltage $V_{L}$ receives low-frequency power from $V_{\mathrm{hv}}$ and highfrequency power from $V_{f}$. As a function of $V_{\mathrm{hv}}$ and $V_{f}$, the load voltage is equal to

$$
\begin{aligned}
& V_{L}(s)= \frac{\omega_{a}}{s+\omega_{a}} V_{\mathrm{hv}}(s)+\frac{C}{C+C_{p}} \frac{s}{s+\omega_{a}} V_{f}(s) \quad \text { where, } \\
& \omega_{a}=\frac{1}{R\left(C+C_{p}\right)} .
\end{aligned}
$$

That is, the response from the high-voltage side is a low-pass filter, while the response from the low-voltage side is a highpass filter with attenuation. A key observation is that the filters $\omega_{a} /\left(s+\omega_{a}\right)$ and $s /\left(s+\omega_{a}\right)$ are complementary, i.e., if the attenuation due to $C$ and $C_{p}$ is accounted for, a signal applied to both amplifiers will be perfectly reproduced across the load. Low frequency power is supplied by $V_{\mathrm{hv}}$, while high-frequency power is supplied by $V_{f}$, which is exactly the situation desired.

A drawback of the dual amplifier is that the high-voltage stage must drive two capacitors $C+C_{p}$ rather than only $C_{p}$. As $C$ is chosen around ten times $C_{p}$ the high-voltage stage is effectively reduced in bandwidth by a factor of 11 .

A design issue for the circuit shown in Fig. 2 is the choice of $R$ and $C$. Choosing $C$ is straight forward. $C$ should be chosen so that the ratio $C / C+C_{p}$ is close to unity and that the combination of $C$ and $C_{p}$ does not unnecessarily load the high-voltage stage. A reasonable compromise is $C=10 C_{p}$. With $C$ fixed, the selection of $R$ controls the low and highpass cutoff frequencies. A good choice is to design the cutoff 
frequency and $R$ so that the high-voltage amplifier can be fully utilized, that is, so that at the cutoff frequency, the high-voltage amplifier is about to reach current limit. If the peak-to-peak voltage and current from the high-voltage stage is $V_{\mathrm{p}-\mathrm{p}}$ and $I_{\mathrm{p}-\mathrm{p}}$, the corresponding load impedance $Z$ is

$$
\begin{aligned}
& \frac{V_{\mathrm{p}-\mathrm{p}}}{I_{\mathrm{p}-\mathrm{p}}}=Z=\left|R+\frac{-j}{2 \pi f\left(C+C_{p}\right)}\right|, \\
& \frac{V_{\mathrm{p}-\mathrm{p}}}{I_{\mathrm{p}-\mathrm{p}}}=\sqrt{R^{2}+\frac{1}{4 \pi^{2} f^{2}\left(C+C_{p}\right)^{2}}} .
\end{aligned}
$$

The frequency where this occurs can be set to the filter cutoff frequency by substituting $f=\omega_{a} / 2 \pi$. Simplification yields

$$
\frac{V_{\mathrm{p}-\mathrm{p}}}{I_{\mathrm{p}-\mathrm{p}}}=\sqrt{2 R^{2}} .
$$

Hence, $R$ and the cutoff frequency in Hertz $F_{c}$ are

$$
R=\sqrt{\frac{1}{2}\left(\frac{V_{\mathrm{p}-\mathrm{p}}}{I_{\mathrm{p}-\mathrm{p}}}\right)^{2}} \quad F_{c}=\frac{1}{2 \pi R\left(C+C_{p}\right)} .
$$

\section{B. Range considerations}

Although the low-voltage stage significantly improves high-frequency performance, it is important to note that the penalty is reduced range at high frequencies. However, in many applications this does not present a significant drawback as there is no requirement to drive the actuator at full range above $100 \mathrm{kHz}$. Indeed, the majority of piezoelectric stack actuators would be destroyed by inertial forces and dielectric heating.

The full voltage range of the amplifier can only be realized within the bandwidth of the high-voltage stage, i.e., from dc to $\omega_{a} / 2 \pi \mathrm{Hz}$. More precisely, the full voltage range is reduced by $1 / \sqrt{2}$ at $\omega_{a} / 2 \pi \mathrm{Hz}$.

To avoid saturation of the low-voltage stage in the frequency band where full range is available, the additional first-order complementary filters $G_{H P}(s)$ and $G_{L P}(s)$ shown

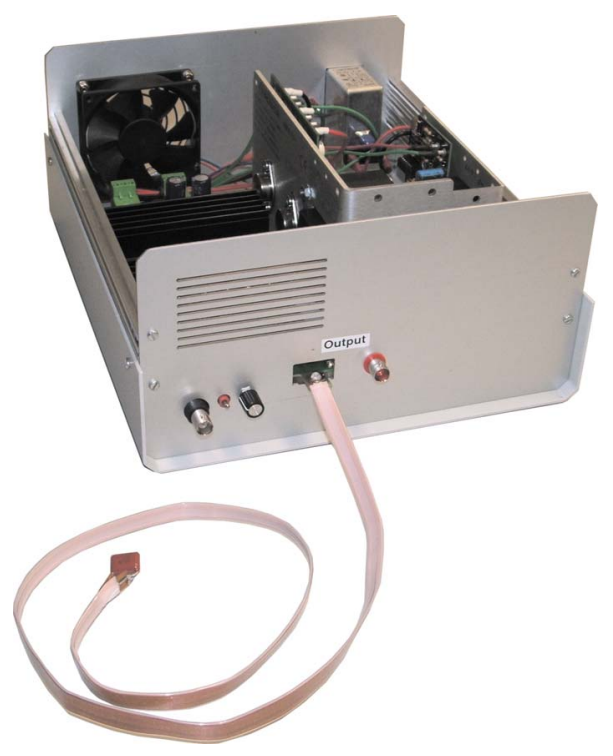

FIG. 3. (Color online) Amplifier enclosure with connected cable and $100 \mathrm{nF}$ capacitive load. in Fig. 2 are required. While $G_{H P}(s)$ removes low frequency signal content to avoid saturation of the low voltage stage, $G_{L P}(s)$ ensures that both stages remain complementary.

The cutoff frequency is determined by the difference in range between the high-and low-voltage stages. $G_{H P}(s)$ should be high enough in frequency to ensure that the range of the low-voltage stage is not exceeded at $\omega_{a} / 2 \pi \mathrm{Hz}$. For example, if the low-voltage stage has one-tenth the range of the high-voltage stage, the cutoff frequency should be ten times greater than the $R C$ cutoff in Eq. (12), i.e.,

$$
G_{H P}(s)=\frac{s}{s+\omega_{b}} \quad \text { where } \omega_{b}=10 \omega_{a} .
$$

Once $G_{H P}(s)$ is decided, the filter $G_{L P}(s)$ is calculated to maintain complimentary signal paths through the low- and high-voltage stages. It is easily verified that this condition is satisfied when

$$
\begin{aligned}
G_{L P}(s) & =\frac{s+\omega_{a}}{a}\left(1-\frac{s}{s+\omega_{b}} \frac{s}{s+\omega_{a}}\right) \\
& =\frac{\left(\omega_{b}+\omega_{a}\right)}{\omega_{a}} \frac{s+\frac{\omega_{b} \omega_{a}}{\omega_{b}+\omega_{a}}}{s+\omega_{b}} .
\end{aligned}
$$

The output range versus frequency of the dual amplifier can be estimated from the dominant poles in the system and the range of the low- and high-voltage stages. That is,

$$
\operatorname{Range}(\omega)=\left|\frac{\omega_{a}}{s+\omega_{a}} R_{\mathrm{HV}}+\frac{s}{s+\omega_{b}} R_{\mathrm{LV}}\right|_{s=j \omega},
$$

where $R_{\mathrm{HV}}$ and $R_{\mathrm{LV}}$ are the full output voltage ranges of the high- and low-voltage stages.

\section{ELECTRICAL DESIGN}

\section{A. High-voltage stage}

The high-voltage stage is a basic voltage-feedback amplifier constructed from an Apex Microtechnology PA98 450 $\mathrm{V}$ opamp. This is supplied by an International Power IHB200-0.12 $215 \mathrm{~V}$ power supply with a current rating of $120 \mathrm{~mA}$. With sufficient storage capacitance, the $120 \mathrm{~mA}$ output current is sufficient to supply the PA98 with its required $20 \mathrm{~mA}$ quiescent current and allow for an amplifier

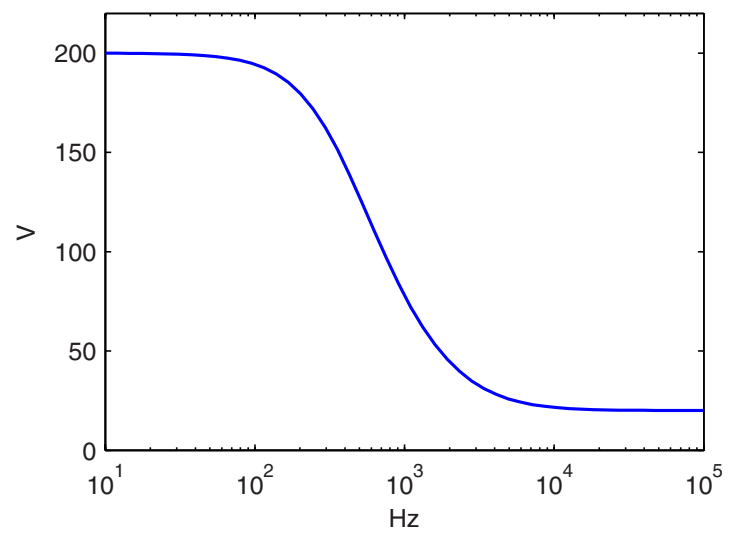

FIG. 4. (Color online) The peak-to-peak output voltage range of the dual amplifier. 


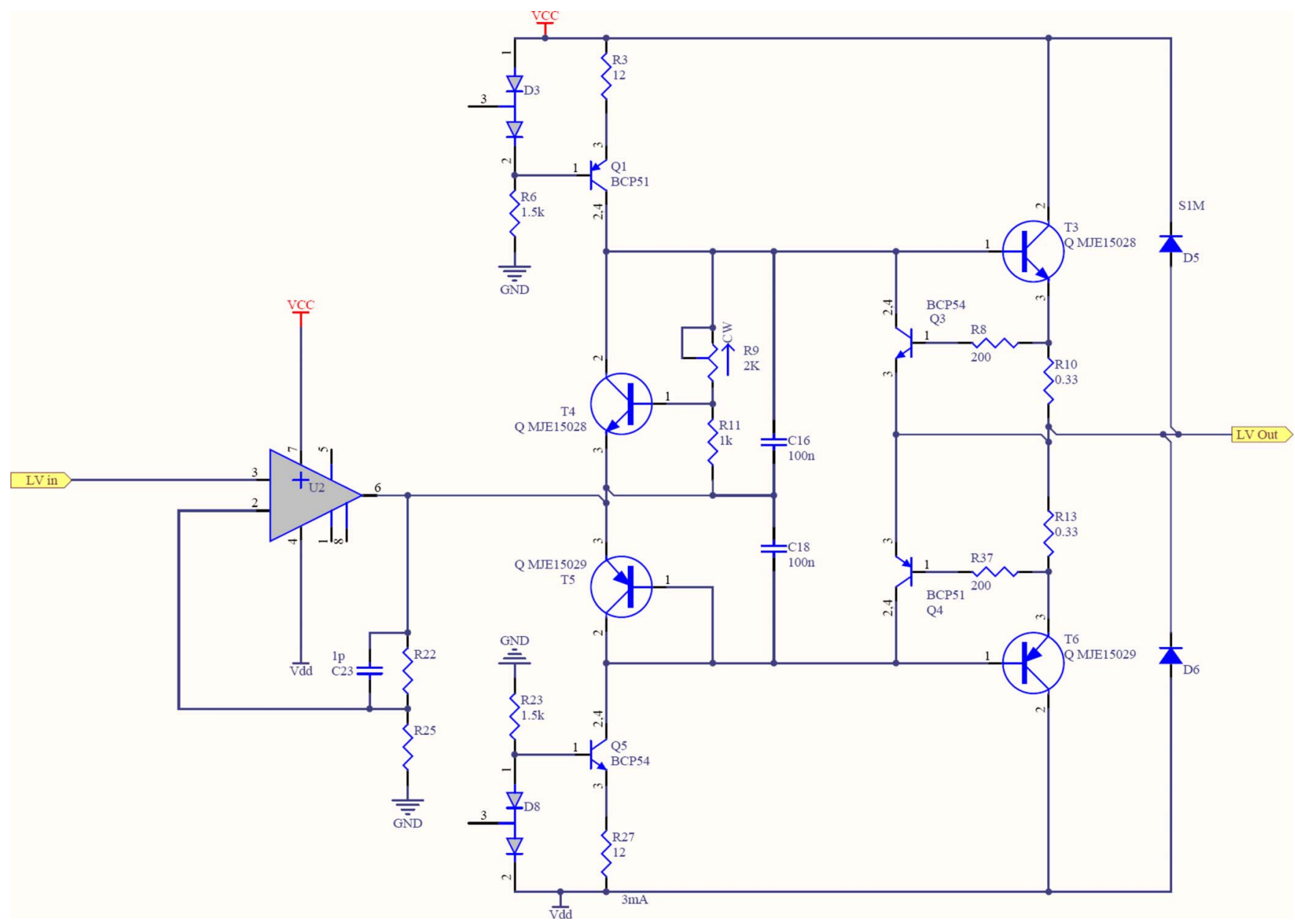

FIG. 5. (Color online) Schematic of the low-voltage amplifier stage. This stage comprises an opamp amplifier to achieve the correct signal gain followed by a bipolar class $\mathrm{AB}$ output.

current limit of $\pm 200 \mathrm{~mA}$ so long as the load is capacitive. The worst case power dissipation into a capacitive load is 20 $\mathrm{W}$, safely within the rated limit of $30 \mathrm{~W}$.

As discussed in Sec. III A, a load capacitance of $100 \mathrm{nF}$ requires a filter capacitance $C$ of approximately $1 \mu \mathrm{F}$. $R$ can be calculated from Eq. (16) as $353 \Omega$. The resulting filter cutoff frequency is $409 \mathrm{~Hz}$. To avoid saturation of the lowvoltage stage, which has only one-tenth the high-voltage range, the filter cutoff frequency of $G_{H P}(s)$ should be approximately $5 \mathrm{kHz}$, i.e., $\omega_{b}=2 \pi 5000$. The resulting range versus frequency is plotted in Fig. 4.

\section{B. Low-voltage stage}

The necessary amplifier performance requirements for achieving high bandwidth with capacitive loads were identified in Secs. II A and II B. With a $100 \mathrm{nF}$ load, a $1 \mathrm{MHz}$ bandwidth requires an output impedance of less than $1 \Omega$ and a small-signal bandwidth well in excess of $1 \mathrm{MHz}$.

With high-voltage supplies and metal-oxide semiconductor field-effect output transistors, low output impedance is extremely difficult to achieve. However, due to the reduced supply voltage, high-performance bipolar transistors can be utilized. These transistors can be operated under heavy forward bias to drop the output impedance to well below $1 \Omega$. A bias current of $100 \mathrm{~mA}$ was found to be sufficient.
The schematic of a wide-bandwidth low-voltage amplifier is shown in Fig. 5. This circuit utilizes a high-speed opamp to achieve the gain of 21 and bandwidth in excess of $10 \mathrm{MHz}$. The following biasing stage provides drive for the output transistors and protects the opamp from excessive current load. The output transistors are high-performance audio types and provide excellent linearity in open-loop. With sufficient bias, the output stage is highly linear and does not require global voltage feedback. This is a major advantage as the gain stage is not limited in bandwidth by the pole formed by the output impedance and load capacitance.

A further advantage of the low-voltage stage is that it can provide large output currents at high frequency without major power dissipation. For example, a $300 \mathrm{kHz} 20 \mathrm{Vp}-\mathrm{p}$ sine wave with $100 \mathrm{nF}$ load requires 1.9 A peak current. With a 2 A current limit, the worst-case power dissipation is around $30 \mathrm{~W}$. In comparison, a $200 \mathrm{~V}$ amplifier would dissipate $400 \mathrm{~W}$ in the same scenario, which is highly impractical.

With the high- and low-voltage stages complete, the assembled dual amplifier was tested for frequency response and drive capability with a $100 \mathrm{nF}$ load. In Fig. 6, the amplifier's response to a $300 \mathrm{kHz} 20 \mathrm{Vp}-\mathrm{p}$ sine wave is plotted. The phase lag between input and output is extremely low. 


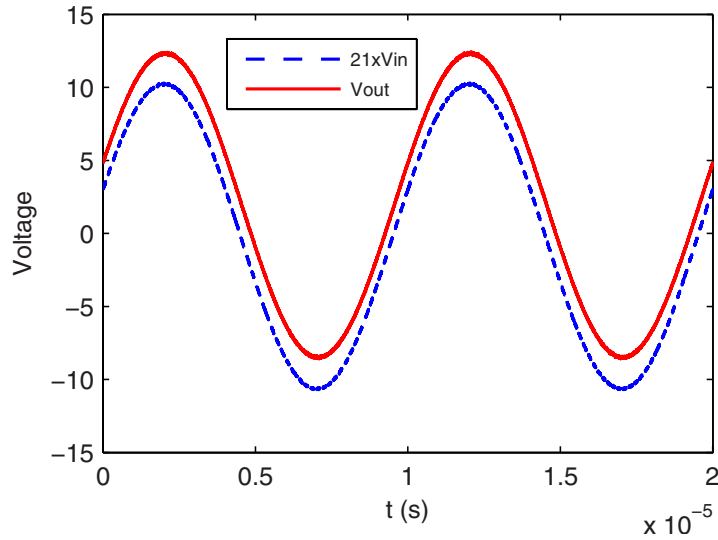

FIG. 6. (Color online) Measured amplifier input and output with a $300 \mathrm{kHz}$ sine wave reference and $100 \mathrm{nF}$ load. The peak-to-peak current is $3.8 \mathrm{~A}$

\section{Cabling and interconnects}

In Sec. II A the cable inductance was identified as a major limitation of amplifier bandwidth when driving highly capacitive loads. As cable inductance increases with the distance between the two conductors, it is desirable to position the conductors as closely together as possible. Simply using twisted small diameter wire is not sufficient as the resistance of the conductors is also of importance. A better solution is to use copper foil for each conductor separated by a thin insulating layer, this configuration yields low inductance, low resistance, and a low characteristic impedance (1).

Cables with the geometry discussed above have been developed for audio applications, one manufacturer is Alphacore. The MI- 2 cable pictured in Figs. 3 and 7 contains two copper foil conductors, a Polyester dielectric and a Polycarbonate outer layer. The width and thickness is 9.5 $\times 0.254 \mathrm{~mm}$ which provides a satisfactory resistance of $14 \mathrm{~m} \Omega$ per meter. Thanks to the high dielectric strength of the insulator, the conductors are separated by only $76 \mu \mathrm{m}$ which results in a cable inductance of only $33 \mathrm{nH}$ per meter, approximately an order of magnitude less than RG-58 coaxial cable (which is $250 \mathrm{nH} / \mathrm{m}$ ).

The inductance of connectors between the cable and amplifier is also of importance. In this work, to minimize loop area, the cable is connected directly to the amplifier circuit board by bolting or soldering it to the exposed traces on the top and bottom surfaces. This configuration is pictured in

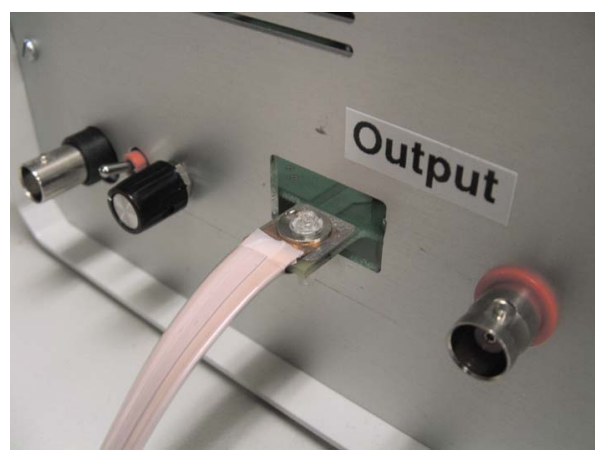

FIG. 7. (Color online) Close-up of low-inductance connection between amplifier circuit board and cable.
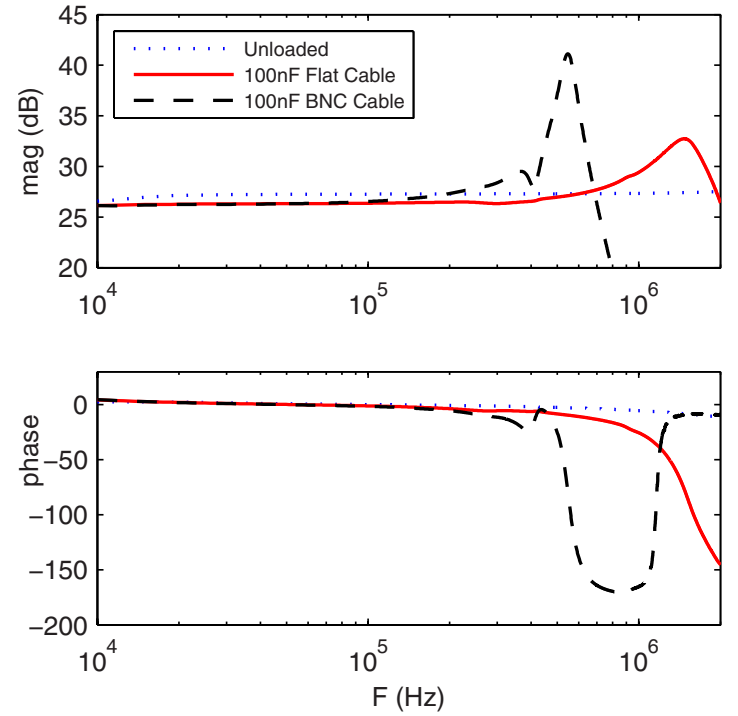

FIG. 8. (Color online) Amplifier frequency response with open-circuit and capacitive loads.

Fig. 7, where a nylon bolt is used to fix the cable onto the PCB.

The frequency response from amplifier input to load voltage is plotted in Fig. 8. With no load, the amplifier bandwidth is exceptional at around $8 \mathrm{MHz}$. With a $100 \mathrm{nF}$ load and a standard $50 \Omega$ coaxial cable, a cable resonance appears at $350 \mathrm{kHz}$. In contrast, the resonance frequency with MI-2 cable is $1.5 \mathrm{MHz}$.

\section{CONCLUSIONS}

In this work, the major factors limiting bandwidth in standard piezoelectric drives were identified as:

- high output impedance,

- high cable inductance,

- low small-signal bandwidth due to an additional feedback pole, and

- insufficient current capacity due to high power dissipation.

These limitations were overcome by combining a standard high-voltage amplifier with a fast low-voltage amplifier. Due to the lesser supply voltage of the low-voltage amplifier, it was possible to utilize a heavily biased bipolar output stage to provide low output impedance. The low supply voltage also allows an order of magnitude increase in output current with no significant increase in power consumption or heat dissipation.

Aside from the amplifiers output impedance and smallsignal bandwidth, the cable and interconnect inductance proved to be the greatest limitation to bandwidth. A flat foil cable with ultralow inductance is required to achieve maximum bandwidth.

The completed amplifier had a low-frequency range of $200 \mathrm{Vpk}$, a high-frequency range of $30 \mathrm{Vp}-\mathrm{p}$, and a smallsignal bandwidth of over $2 \mathrm{MHz}$ with a $100 \mathrm{nF}$ load capacitance. The main bandwidth limitation was a cable resonance at $1.5 \mathrm{MHz}$. The device was demonstrated to drive a $100 \mathrm{nF}$ load capacitance with a $300 \mathrm{kHz} 20 \mathrm{Vp}$-p sine wave with 
negligible phase lag. Although the peak-to-peak current was $3.8 \mathrm{~A}$, the worst case power dissipation was only $30 \mathrm{~W}$. The drawbacks of the dual amplifier are reduced range at high frequencies and a slower high-voltage response. Present work involves incorporating the amplifier into a high-speed atomic force microscope with positioning and force control bandwidths exceeding $100 \mathrm{kHz}$.

\section{ACKNOWLEDGMENTS}

This research was supported by an Australian Research Council Discovery Project (Contract No. DP0986319).

${ }^{1}$ A. A. Tseng, A. Notargiacomob, and T. P. Chen, J. Vac. Sci. Technol. B 23, 877 (2005)

${ }^{2}$ K. Uchino and J. R. Giniewica, Micromechatronics (Dekker, New York, 2003).

${ }^{3}$ Scanning Probe Microscopy and Spectroscopy. Theory, Techniques, and
Applications, 2nd ed., edited by D. Bonnell (Wiley-VCH, Hoboken, NJ, 2001).

${ }^{4}$ A. J. Fleming and K. K. Leang, Ultramicroscopy 108, 1551 (2008).

${ }^{5}$ A. Preumont, Mechatronics, Dynamics of Electromechanical and Piezoelectric Systems (Springer, New York, 2006).

${ }^{6}$ S. S. Aphale, A. J. Fleming, and S. O. R. Moheimani, Smart Mater. Struct. 16, 439 (2007).

${ }^{7}$ G. E. Fantner, P. Hegarty, J. H. Kindt, G. Schitter, G. A. G. Cidade, and P. K. Hansma, Rev. Sci. Instrum. 76, 026118 (2005).

${ }^{8}$ H. Yamashita, N. Kodera, A. Miyagi, T. Uchihashi, D. Yamamoto, and T. Ando, Rev. Sci. Instrum. 78, 083702 (2007).

${ }^{9}$ M. J. Rost, L. Crama, P. Schakel, E. van Tol, G. B. E. M. van VelzenWilliams, C. F. Overgauw, H. ter Horst, H. Dekker, B. Okhuijsen, M. Seynen, A. Vijftigschild, P. Han, A. J. Katan, K. Schoots, R. Schumm, W. van Loo, T. H. Oosterkamp, and J. W. M. Frenken, Rev. Sci. Instrum. 76, 053710 (2005).

${ }^{10}$ K. K. Leang and A. J. Fleming, Asian Journal of Control 11, 144 (2009).

${ }^{11}$ H. Müller, Rev. Sci. Instrum. 76, 084701 (2005).

${ }^{12}$ H. Müller, S. Chiow, Q. Long, and S. Chu, Opt. Lett. 31, 202 (2006).

${ }^{13} \mathrm{P}$. Horowitz and W. Hill, The Art of Electronics (Cambridge University Press, Cambridge, 1989). 